\title{
Diseño de una actividad lúdica para enseñanza del problema de inventario de una sola vez con teoría de la decisión: Abastece la tropa
}

Design of playful activity for teaching the newsboy inventory problem with decision theory: Supply the troop

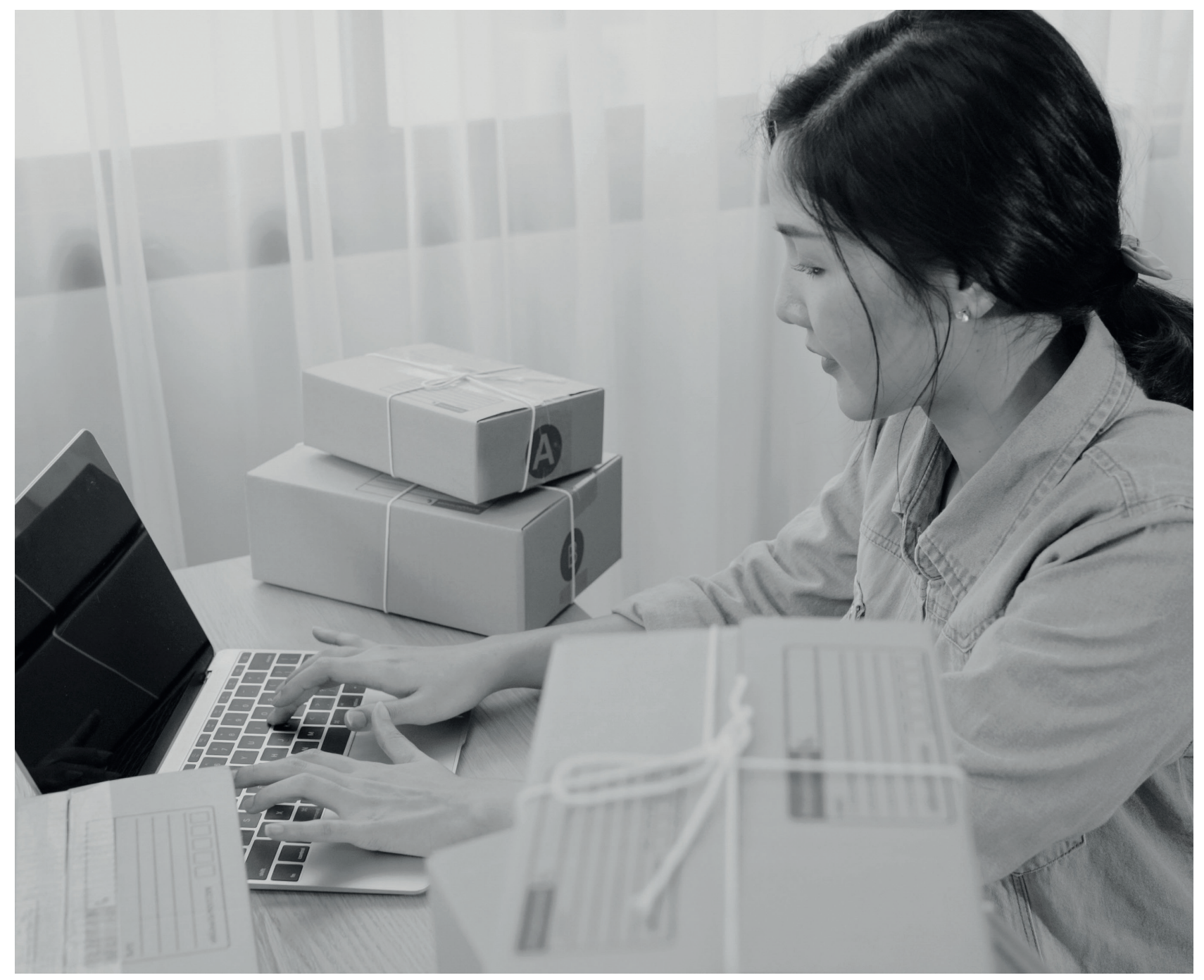




\title{
Diseño de una actividad lúdica para enseñanza del problema de inventario de una sola vez con teoría de la decisión: Abastece la tropa $^{1}$
}

\author{
Design of playful activity for teaching the newsboy inventory \\ problem with decision theory: Supply the troop
}

\author{
Jair Eduardo Rocha-González², Cristian David Quintero-Romero³, Walter Stiven Santamaria-Melo4
}

Artículo recibido en abril 28 de 2020; artículo aceptado en septiembre 8 de 2020

\begin{abstract}
Este artículo puede compartirse bajo la Licencia Creative Commons Atribución-NoComercial-Compartirlgual 4.0 Internacional y se referencia usando el siguiente formato: Rocha-González, J. E., Quintero-Romero, C. D. y Santamaria-Melo, W. S. (2021). Diseño de una actividad lúdica para enseñanza del problema de inventario de una sola vez con teoría de la decisión: Abastece la tropa. I+D Revista de Investigaciones, 16(1), 147-164. http://dx.doi.org/10.33304/revinv.v16n1-2021013.
\end{abstract}

\section{Resumen}

En diferentes tipos de organización la estimación del tamaño de lotes de compra o producción es uno de los problemas frecuentes en la planeación de las cadenas de suministro, en las cuales participan estas unidades productivas. Esto se ha denominado el modelo de inventario de una sola vez o de "vendedor de periódicos". De acuerdo con este principio, se realiza el diseño de una actividad lúdica aplicando la metodología cibernética de tercer orden, para facilitar el proceso de enseñanza-aprendizaje de un sistema, donde la estimación del tamaño de lote para compra o fabricación se centra en un artículo perecedero o con temporada de comercialización reducida, mediante un método inspirado en la teoría de la decisión. El escenario lúdico sucede en una tropa del ejército, en donde la enseñanza y el aprendizaje de conceptos y métodos de solución al problema se produce a través de la simulación de condiciones como demanda estocástica, con ciclos de producto y comercialización reducidos en un lapso de planeación para un artículo.

Palabras clave: Demanda estocástica, juego de simulación, inventario, toma de decisiones, vendedor de periódico.

\section{Abstract}

In different types of organizations, the estimation of the size of purchase or production lots is one of the frequent problems in the planning of the supply chains in which these productive units participate. This has been called the

\footnotetext{
${ }^{1}$ Artículo de investigación, de enfoque cualitativo, resultado del proyecto culminado relacionado con investigación de lúdicas del semillero de investigación IMAPRO, perteneciente al área de ciencias sociales e ingeniería industrial, subárea de educación en ingeniería, desarrollado por el grupo investigación Glll, financiado por la Universidad de La Salle (Bogotá D. C., Colombia). Dirección: calle 2 n. ${ }^{\circ}$ 10-70. PBX: +5713535360. Fecha de inicio: 10 de julio de 2019. Fecha de terminación: 29 de febrero de 2020.

${ }_{2}^{2}$ Maestría en Ingeniería Industrial, Universidad Francisco José de Caldas. Vinculado al grupo de investigación GIII, Universidad de La Salle (Bogotá D. C., Colombia). Dirección: calle 2 n. 10-70. PBX: +5713535360. ORCID ID: https://orcid.org/0000-0003-4636-5462. Correo electrónico institucional: jerocha@unisalle.edu.co.

${ }^{3}$ Ingeniero industrial, Universidad de La Salle. Vinculado al grupo de investigación GIII, Universidad De La Salle (Bogotá D. C., Colombia). Dirección: calle 2 n. ${ }^{\circ} 10-70$. PBX: +5713535360. ORCID ID: https://orcid.org/0000-0001-7127-8892. Correo electrónico institucional: Cquintero06@unisalle.edu. co.

${ }^{4}$ Ingeniero industrial, Universidad de La Salle. Vinculado al grupo de investigación GIII, Universidad de la Salle (Bogotá D. C., Colombia). Dirección: calle 2 n. ${ }^{\circ}$ 10-70. PBX: +5713535360. ORCID ID https://orcid.org/0000-0001-7859-9664. Correo electrónico institucional: wsantamaria80@unisalle. edu.co.
} 
Jair Eduardo Rocha-González, Cristian David Quintero-Romero, Walter Stiven Santamaria-Melo Diseño de una actividad lúdica para enseñanza del Problema de Inventario de Una Sola Vez con Teoría de la Decisión: Abastece la tropa

one-time inventory or "newsagent" model. According to this principle, a playful activity is designed to facilitate the teaching-learning process of a system where the estimation of the lot size for purchase or production is focused on a perishable item or an item with a reduced marketing season, through a method inspired by decision theory. The playful scenario takes place in an army troop, where the teaching and learning of concepts and methods of solution to the problem occurs through the simulation of conditions such as stochastic demand with reduced product and marketing cycles in a planning period for an article.

Keywords: Stochastic demand, simulation game, inventory, decision making, newspaper salesman.

\section{Introducción}

La estimación de tamaños de lote para situaciones de fabricación o distribución de productos es en esencia uno de los propósitos de la gestión de inventarios. Este proceso se realiza teniendo como fundamento la naturaleza de la demanda, el ciclo de vida útil del artículo y los costos e impacto ocasionados en la gestión logística y, por ende, en la toma de decisiones logísticas (Nevison y Burstein, 1984; Richards, 1976; Sphicas, 1982; Tersine, 1998).

Ante esta perspectiva, una de las situaciones comunes trata del cálculo del tamaño de lote óptimo para un producto perecedero, el cual posee un ambiente de demanda estocástica y un único periodo de abastecimiento, que en la literatura se conoce como el problema de inventario de una sola vez, o Newsboy Problem, por sus siglas en inglés (Van Mieghem y Rudi, 2002).

Un ejemplo real de estimación de tamaño de lote de compra se presenta cuando los ejércitos enfrentan el desafío de comprar y transportar una cantidad suficiente de víveres o insumos, para satisfacer necesidades alimentarias de los integrantes de estos grupos durante recorridos en diferentes ambientes, como son los recorridos en la selva, ríos, montañas, desiertos, entre otros.

Por tanto, la actividad didáctica presentada en este artículo tiene como propósito el diseño de un ambiente lúdico que integre los elementos de cálculo de tamaño de lote y el contexto de las tropas en condiciones difíciles, para ser usado en los procesos de enseñanza y aprendizaje del problema de inventario de una sola vez, bajo un enfoque de teoría de la decisión, siguiendo las pautas metodológicas para el desarrollo de lúdicas para la enseñanza de diferentes temáticas de la ingeniería (Arango Londoño et al., 2014; Rocha-Gonzalez, 2011).

\section{Marco referencial}

El problema de inventario de una sola vez o "vendedor de periódicos" consiste en realizar un único pedido al inicio del periodo de comercialización, fabricación o consumo del producto, con el cual es posible cubrir la demanda estocástica de artículos a corto tiempo a futuro (Bierman et al., 1996).
Este tipo de modelo de abastecimiento se diferencia de los modelos de cantidad fija de pedido en que se realiza un único pedido o lote de fabricación, debido a la corta vida útil del producto o su reducida temporada de comercialización. Otra diferencia sustancial consiste en que los modelos de una sola vez carecen de una demanda fija en el tiempo, factor que incorpora en el análisis a la incertidumbre en el volumen de consumo como elemento sustancial del modelado de este tipo de problemas de suministro (Silver et al., 1998; Sippper y Bulfin, 1998; Xue et al., 2011).

De acuerdo con lo anterior, el modelo para la toma de decisiones del cálculo de tamaño de lote en una sola vez o "vendedor de periódicos" considera la demanda probabilística bajo dos ambientes, los cuales se clasifican de acuerdo con los criterios descritos en la Tabla 1 (Sippper y Bulfin, 1998).

Tabla 1

Clasificación del tipo de demanda estocástica asociada al problema de decisión de una sola vez

\begin{tabular}{cl}
\hline $\begin{array}{c}\text { Tipo de demanda } \\
\text { estocástica }\end{array}$ & \multicolumn{1}{c}{ Características } \\
\hline Discreto & $\begin{array}{l}\text { En este caso se establece un conjunto de valo- } \\
\text { res de ventas que se repiten en un intervalo de } \\
\text { tiempo, para los cuales se estima la frecuencia } \\
\text { de ocurrencia de estas ventas en términos de } \\
\text { una función de probabilidad discreta. }\end{array}$ \\
Continuo & $\begin{array}{l}\text { Para este caso se establecen los diferentes valo- } \\
\text { res de venta que ocurren durante periodos de } \\
\text { tiempo consecutivos de un periodo de planea- } \\
\text { ción, cuyo comportamiento aleatorio es posible } \\
\text { de representar con una función de distribución } \\
\text { de probabilidad continua. }\end{array}$ \\
\hline
\end{tabular}

Fuente: Autores.

A partir de esta clasificación de la demanda estocástica para el problema descrito, es posible en ambos casos determinar el tamaño de lote de compra o fabricación mediante la estimación de una función acumulada de probabilidad, con el uso de métodos como la suma de las probabilidades discretas o la integración numérica (Sippper y Bulfin, 1998; Tersine, 1998).

Una vez estimada la función acumulada de probabilidad para la cantidad de ventas o demanda histórica en el sistema, se calculan los costos unitarios por producto faltante o excedente al tamaño de orden inicialmente realizado. Esto hace referencia a la estimación de los 
Jair Eduardo Rocha-González, Cristian David Quintero-Romero, Walter Stiven Santamaria-Melo

Diseño de una actividad lúdica para enseñanza del Problema de Inventario de Una Sola Vez con Teoría de la Decisión: Abastece la tropa

costos incurridos por una inadecuada gestión en el cálculo del tamaño de lote (Sippper y Bulfin, 1998; Tersine, 1998).

Una vez obtenido el costo unitario por producto faltante o excedente, y la función de distribución de probabilidad que caracteriza el comportamiento de la demanda o ventas del sistema, se realiza el cociente entre los costos unitarios por faltantes y la suma de excedentes y faltantes. Este resultado se iguala a la función de distribución de probabilidad de la demanda, para hallar el tamaño de lote de compra que minimiza las cantidades no deseadas de faltantes y sobrantes (Sippper y Bulfin, 1998; Tersine, 1998).

Un método alternativo para la estimación de tamaños de lote en una sola vez consiste en realizar un análisis de decisión, mediante el cálculo del valor monetario esperado de unidades faltantes y sobrantes, que resultan de las combinaciones posibles entre demanda y pedidos realizados (Bierman et al., 1996).

De esta forma, este método, para el caso de demanda estocástica discreta, inicia con el cálculo de los costos por unidad en caso de ser faltante o excedente una vez ha ocurrido las ventas en el periodo para el cual se toma la decisión, tal como se muestra en las ecuaciones (1) y (2) (Sippper y Bulfin, 1998).

Posterior a ello, se realiza el cálculo de las cantidades esperadas de faltantes o excedentes para cada combinación de valores de venta esperadas y de pedidos posibles a realizar en el mismo rango numérico de la demanda de artículos, mediante un formato tabular, de ser posible (Bierman et al., 1996).

Luego, es posible calcular el costo y utilidad total para cada combinación de pedidos y demanda, al realizar la multiplicación del costo por unidad de faltante o excedente y por la cantidad esperada de faltantes o excedentes, tal como se muestra en la ecuación (3) (Bierman et al., 1996).

$$
\begin{gathered}
\pi=(P v-C)+C P B V \\
C_{0}=C+C_{L O G}-V S \\
U=(P v-C) \times Q^{*}-\left[\left(\pi \times\left(Q^{*}-D\right) \times F(D)\right)+\right. \\
\left.\left(C_{0} \times\left(D-Q^{*}\right) \times F(D)\right)\right]
\end{gathered}
$$

Donde:

Pv: $\quad$ Precio de venta por unidad de producto

$C$ : $\quad$ Costo de venta por unidad de producto

$Q^{*}$ : Tamaño de lote óptimo de pedido

Vs: $\quad$ Valor de salvamento por unidad

$C_{\text {LoG }}$ Costo logístico por unidad por deshacerse de excedentes

CPBV: Costo de pérdida por unidad de buena voluntad

$D-Q^{*}$ : Cantidad esperada de faltantes

$Q^{*}$ - D: Cantidad esperada de sobrantes

En consecuencia, la ecuación (1) representa el costo por unidad faltante que se presenta cuando la demanda es superior a la cantidad pedida, la cual se calcula como la utilidad perdida por unidad no disponible de producto más la perdida de las ventas futuras de un cliente que abandona el servicio (Sippper y Bulfin, 1998).

De manera similar, la ecuación (2) presenta el cálculo del costo por unidad excedente, en caso de un pedido superior a la demanda real, la cual está compuesta por el costo por unidad del producto no vendido más el costo de deshacerse del sobrante menos un valor de salvamento que se puede recuperar en el proceso de eliminación del sobrante por venta del material, entre otras causas (Sippper y Bulfin, 1998).

Por último, la ecuación (3) presenta la forma de estimación de la utilidad total por lote pedido de tamaño $\mathrm{Q}^{*}$, estimado a través de un criterio de decisión y el valor esperado de faltantes y excedentes para las diferentes combinaciones de oferta y demanda en estudio (Sippper y Bulfin, 1998; Tersine, 1998), en relación a los criterios posibles de decisión. Estos son descritos en la Tabla 2, de acuerdo con la literatura acerca del tema (Bierman et al.,

\begin{tabular}{|c|c|c|c|}
\hline Criterio & Características & Expresión matemática & \\
\hline Mayor Probabilidad & $\begin{array}{l}\text { Se selecciona la demanda con mayor probabilidad de ocurrencia como la cantidad a } \\
\text { ordenar o a fabricar }\end{array}$ & $\operatorname{Max}\left[P\left(D_{x}\right)\right]$ & (4) \\
\hline Valor Condicional & $\begin{array}{l}\text { Establece como cantidad a ordenar o a fabricar la máxima utilidad por cada combinación } \\
\text { de pedido y demanda }\end{array}$ & $V C=(P v-C) \times Q$ & (5) \\
\hline Pérdidas esperadas & Establece como cantidad a ordenar o a fabricar la mínima pérdida por errores en la & $D>A \quad d_{f} \times D$ & \\
\hline $\begin{array}{l}\text { Valor Monetario } \\
\text { esperado }\end{array}$ & $\begin{array}{l}\text { Selecciona como valor a ordenar o a fabricar la cantidad esperada de demanda calculada } \\
\text { a partir de la probabilidad y la máxima utilidad }\end{array}$ & $\begin{array}{l}D<A \quad d_{s} \times D \\
V M E=\sum_{D=1}^{x} P\left(D_{x}\right) \times V C_{x}\end{array}$ & (8) \\
\hline \multirow[t]{2}{*}{$\begin{array}{l}\text { Pérdida de } \\
\text { oportunidad } \\
\text { esperada }\end{array}$} & $\begin{array}{l}\text { Selecciona como valor a ordenar o a fabricar la cantidad esperada de demanda calculada } \\
\text { a partir de la probabilidad y la mínima pérdida }\end{array}$ & $P O E=\sum_{D=1}^{x} P\left(D_{x}\right) \times P C_{x}$ & (9) \\
\hline & $\begin{array}{l}\text { VC: Valor Condicional } \\
\text { VME: Valor monetario esperado }\end{array}$ & & \\
\hline
\end{tabular}
1996).

Tabla 2

Criterios utilizados para la estimación del tamaño de lote desde una perspectiva de la teoría de decisiones

Fuente: Autores. 
Jair Eduardo Rocha-González, Cristian David Quintero-Romero, Walter Stiven Santamaria-Melo Diseño de una actividad lúdica para enseñanza del Problema de Inventario de Una Sola Vez con Teoría de la Decisión: Abastece la tropa

Para ello, este artículo comprende los conceptos relevantes para determinar la cantidad óptima del tamaño de un lote de compra o producción por única vez, con el propósito de minimizar las cantidades de faltantes y sobrantes una vez el producto agote su vida útil o termine la temporada (Silver et al., 1998).

Un sobrante o excedente es la unidad que se descarta una vez ha pasado el lapso de tiempo durante el cual se presenta la demanda. En otras palabras, son las unidades perdidas, puesto que no se lograron vender en el periodo esperado de demanda (Tersine, 1998). Un faltante, por otra parte, es la unidad no disponible para su venta durante un horizonte de consumo, lo cual significa que son las unidades que no se incluyeron en un pedido y fueron solicitados por la demanda (Sippper y Bulfin, 1998).

También es importante señalar que la lúdica presentada en este artículo utiliza los conceptos contenidos en la Tabla 3, los cuales tienen relevancia en la presentación de la técnica que se pretende enseñar para la estimación de tamaño de lotes de productos en una sola vez.

Tabla 3

Conceptos utilizados para la estimación del tamaño de lote desde una perspectiva de la teoría de decisiones

\begin{tabular}{|c|c|}
\hline Concepto & Definición \\
\hline Valor condicional & $\begin{array}{l}\text { Descripción de utilidades obtenidas para cada combinación de demanda y pedido a partir de los } \\
\text { registros históricos. }\end{array}$ \\
\hline Pérdida de oportunidad condicional & Suceso que identifica la pérdida de utilidad que se obtiene por no suplir la demanda. \\
\hline Valor monetario esperado & Representación monetaria del valor esperado multiplicado por su probabilidad de suceso. \\
\hline Pérdida de oportunidad esperada & Total, pérdida de utilidad según la ocurrencia o probabilidad de la demanda. \\
\hline Valor esperado de la información perfecta & $\begin{array}{l}\text { Valor óptimo de utilidad según la demanda adecuada para cada día, esto por la probabilidad del } \\
\text { suceso. }\end{array}$ \\
\hline Árbol de decisión & $\begin{array}{l}\text { Descripción de sucesos partiendo de todas las posibles acciones o pedidos con sus respectivas } \\
\text { probabilidades mediante líneas de conexión. }\end{array}$ \\
\hline Costo de inventario & Representa el costo de excedentes o faltantes por periodo según el suceso. \\
\hline
\end{tabular}

Fuente: Autores, con base en Bierman et al. (1996).

Ahora bien, partiendo de la concepción de actividad lúdica como actividad planificada para recrearse, entretenerse y divertirse, dejando un aprendizaje (Arango Londoño et al., 2014), en la lúdica se aclaran las definiciones técnicas del manejo de inventarios para demandas estocásticas en productos de temporada con teoría de la decisión establecidas en el marco referencial construido para este fin.

En este sentido, el diseño de esta lúdica se conecta con el proceso de enseñanza al actuar en la manera de dictar la construcción de conocimientos y saberes relacionados, bajo un contexto de la vida cotidiana como lo es el Ejército de Colombia, respetando los espacios y situaciones de tiempo determinados en el mismo contexto. Adicionalmente, es importante relacionar el aprendizaje particular, en donde destaca el desarrollo de estructuras cognitivas, modificando los sistemas operativos para entender las situaciones y problemas de cada día.

\section{Metodología}

Este trabajo es de tipo cualitativo-descriptivo, que busca implementar estrategias didácticas en el proceso de enseñanza y aprendizaje de los diferentes conceptos de la ingeniería industrial en la educación superior.
El diseño de la actividad lúdica propuesta tiene como elemento metodológico el desarrollo de una simulación didáctica siguiendo los fundamentos de cibernética de tercer orden para la formulación de juegos, tal como se describe en el texto de la Red Iddeal presentado en el año 2014 (Rocha González et al., 2014). Con esto se concibe esta actividad como un sistema retroalimentado que integra diferentes tipos de recursos.

De este modo, la metodología utilizada contiene cuatro niveles de actuación y pensamiento humano, como se describe en la Figura 1, en la cual la relación de elementos de entradas, transformación y salidas conforman un nivel ontológico referido a la realización de la actividad lúdica. De forma similar, los elementos de planeación y criterios establecen el segundo nivel de pensamiento, en el cual se identifica el aprendizaje de los conceptos presentados en un proceso epistemológico.

Los elementos de propósito y metas establecen el tercer nivel de actuación y pensamiento en un conjunto semiológico, el cual consiste en el tratamiento de la información involucrado en el juego y el cómo se refleja dicha información para el aprendiz. Finalmente, los elementos de valores en uso y declarado conforman un nivel axiológico, en el cual es posible establecer las reglas sociales e individuales que rigen la actuación de los participantes en la lúdica en cuestiones no declaradas en la situación en análisis (Rocha González et al., 2015). 
Jair Eduardo Rocha-González, Cristian David Quintero-Romero, Walter Stiven Santamaria-Melo

Diseño de una actividad lúdica para enseñanza del Problema de Inventario de Una Sola Vez con Teoría de la Decisión: Abastece la tropa

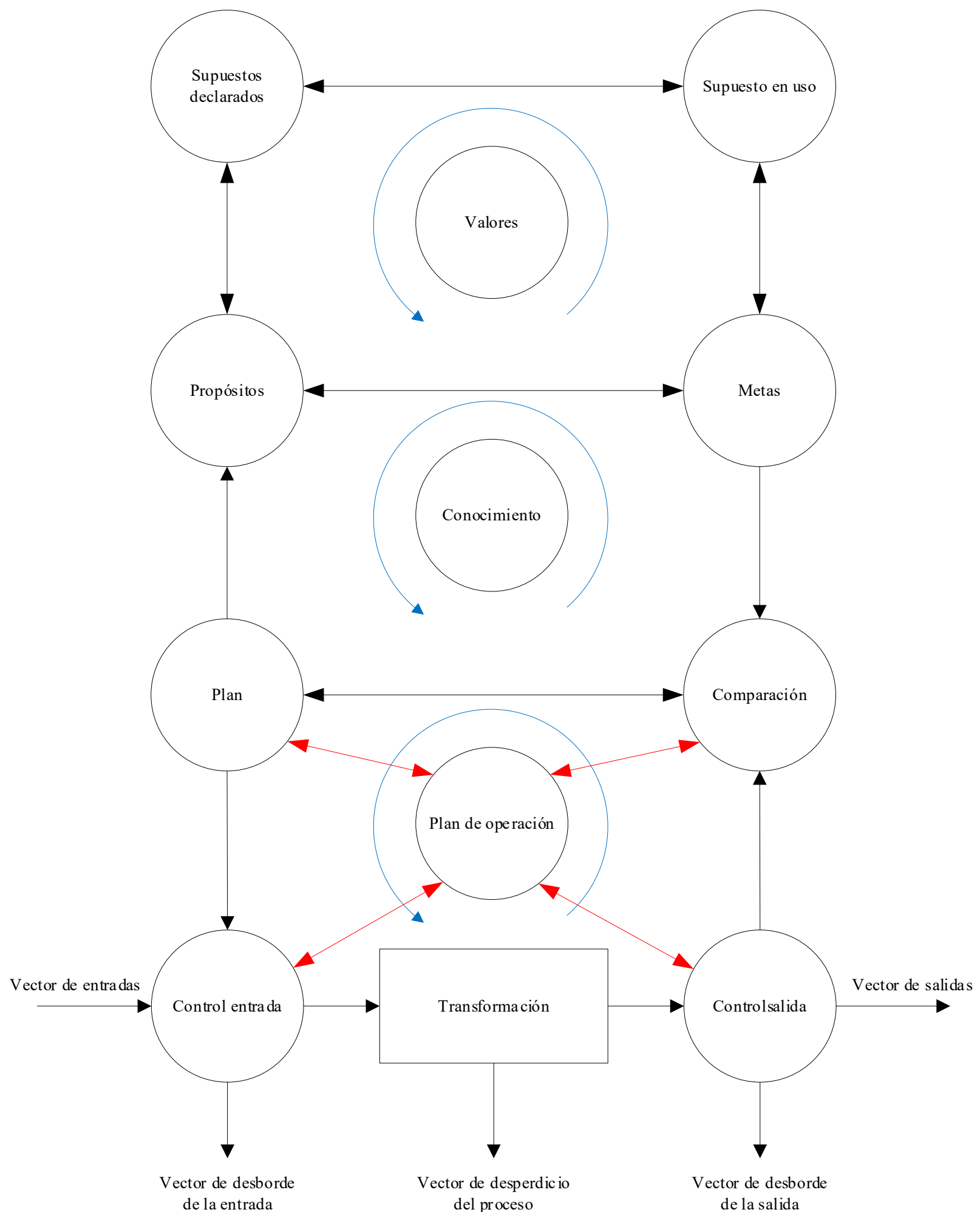

Figura 1. Círculo de retroalimentación de tercer orden genérico. Fuente: Nova Arevalo et al. (2011) citado en Rocha González et al. (2015).

A partir de los conceptos anteriores de cibernética de tercer orden, la actividad lúdica propuesta tiene los elementos presentados en la Figura 2, los cuales describen de manera general los niveles ontológico, epistemológico, semiológico y axiológico. 
Jair Eduardo Rocha-González, Cristian David Quintero-Romero, Walter Stiven Santamaria-Melo Diseño de una actividad lúdica para enseñanza del Problema de Inventario de Una Sola Vez con Teoría de la Decisión: Abastece la tropa

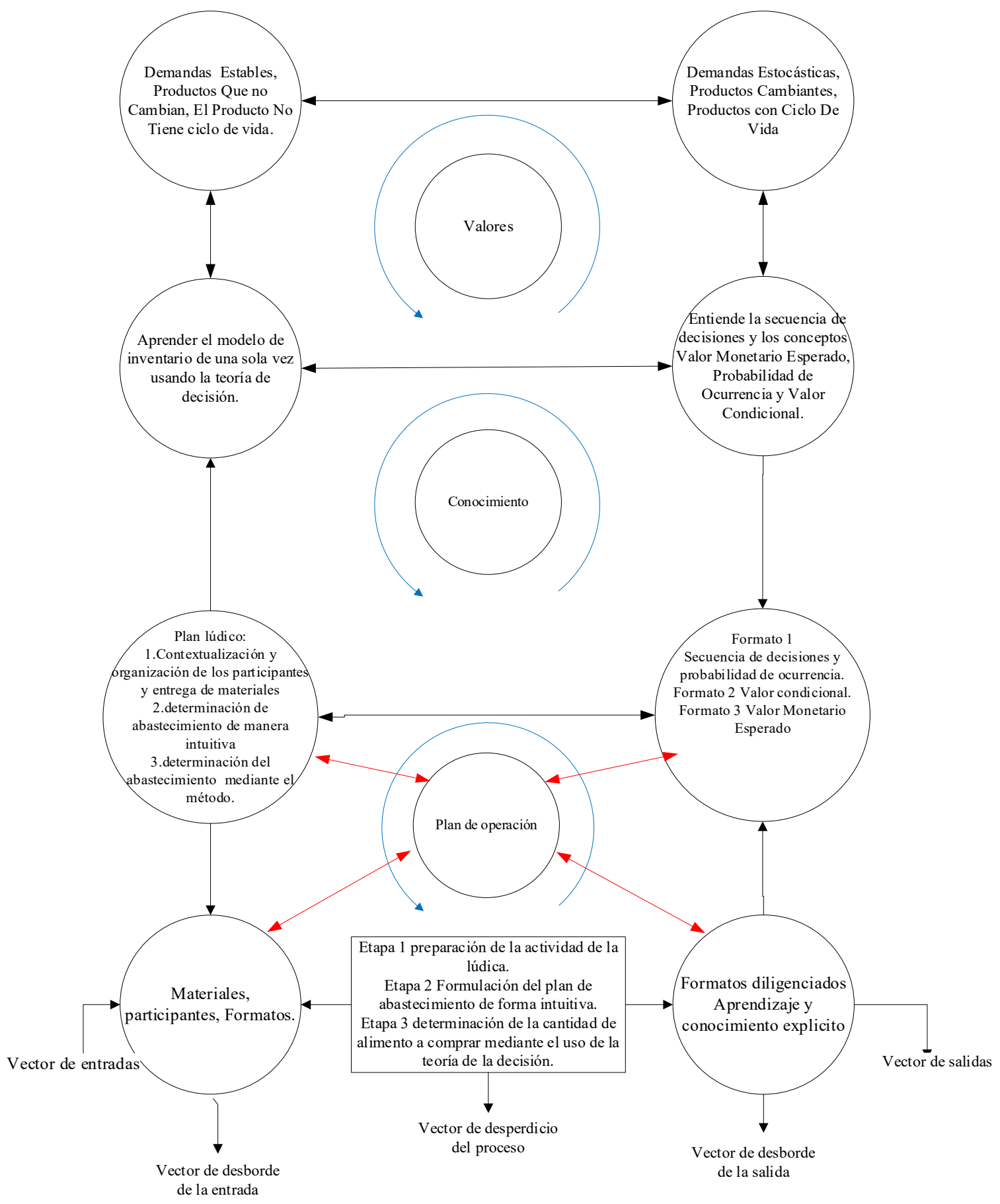

Figura 2. Círculo de retroalimentación de tercer orden de la lúdica. Fuente: Autores.

De acuerdo con lo anterior, estos elementos tienen una descripción más amplia de nivel superior a inferior e izquierda a derecha, de la siguiente manera:

1. Supuesto declarado: Son los referentes de partida para la iniciación del diseño y desarrollo lúdico, en los cuales se presume que las demandas para todo producto es regular y pronosticable, además de considerar que cada producto no tiene cambios en el tiempo y vida útil de largo aliento.

2. Supuesto en uso: Son los referentes de partida que se incluyen en el desarrollo de la actividad lúdica; están compuestos por el comportamiento estocástico de 
Jair Eduardo Rocha-González, Cristian David Quintero-Romero, Walter Stiven Santamaria-Melo

Diseño de una actividad lúdica para enseñanza del Problema de Inventario de Una Sola Vez con Teoría de la Decisión: Abastece la tropa

la demanda, con productos cambiantes y de ciclo de vida corto o por temporada.

3. Propósitos: La finalidad de la lúdica es que los participantes aprendan a proporcionar un plan de abastecimiento mediante el uso de teoría de la decisión en un problema de inventario de una sola vez.

4. Metas: Estas se logran al disgregar el propósito de la lúdica en el aprendizaje de conceptos como Valor Monetario Esperado (VME), Probabilidad de Ocurrencia (PO) y Valor Condicional (VC), los cuales serán medibles en los cálculos realizados en los formatos diligenciados.

5. Plan: El plan lúdico comprende las siguientes etapas: 1) contextualización y organización de los participantes junto con la entrega de materiales; 2) determinación de abastecimiento de manera intuitiva, y 3) determinación del abastecimiento mediante el método de teoría de decisión.

6. Comparación: En este control se establece la apropiación de los conceptos propuestos como metas de aprendizaje, a través de la comparación de la utilización de estos en una situación práctica y los formatos diligenciados resultados de la actividad lúdica. El formato 1 mide la comprensión de la secuencia de decisiones y el cálculo y significado de la probabilidad de ocurrencia; para el formato 2 se establece el grado de apropiación del concepto de cálculo y significado del valor condicional; por último, el formato 3 establece el aprendizaje del método valor monetario esperado con el cual se da solución al problema de inventario de una sola vez.

7. Control de entradas: En este nodo se identifican los recursos lúdicos con los cuales comienza el desarrollo lúdico; es decir, los participantes, los materiales, los espacios y los formatos en los cuales se anotará la información de cada grupo de participantes.

8. Transformación: Este representa la realización de las tres etapas lúdicas. La primera es la preparación de la actividad de la lúdica; la segunda consiste en la formulación del plan de abastecimiento de forma intuitiva; y la tercera es la determinación de la cantidad de alimento por comprar mediante el uso de la teoría de la decisión.

9. Control de salidas: En este nodo se aprecian dos elementos, uno asociado a la comprensión de los conceptos mediante el diligenciamiento de los formatos; otro, el aprendizaje mediante la diversión, proporcionada por la actividad y establecida como significancia y satisfacción.
Una vez se realiza el diseño ya reseñado, se realiza un ejercicio de validación con el propósito de evaluar la coherencia y cohesión del contexto lúdico, el diseño, los formatos y las secuencias de actividades diseñadas.

Para ello, se realizaron cinco sesiones de aplicación de la lúdica con dos grupos de tres estudiantes del semillero de investigación, que pertenecen a los programas de ingeniería industrial e ingeniería en automatización de una universidad de Bogotá. Al finalizar cada sesión, estos estudiantes establecieron los ajustes necesarios de la lúdica considerando los conceptos de teoría de la decisión que previamente eran explicados por un docente del área de investigación de operaciones y consignados en un formato que tenía la misma estructura del diseño presentes en la figura 2 de este documento. Allí era posible realizar una retroalimentación de posibles diferencias entre lo realizado en la lúdica y el concepto esperado de aprendizaje.

\section{Resultados}

\section{Participantes de la lúdica}

Para un buen desarrollo de la actividad lúdica es necesario contar con dos tipos de recurso humano, los cuales son clasificados como orientadores y jugadores.

Los jugadores tendrán que integrar grupos de trabajo de 3 personas, donde cada uno personificará un rol descrito en la Tabla 4.

Tabla 4

Cantidad de personas para jugar

\begin{tabular}{ccl}
\hline Persona & Cantidad & \multicolumn{1}{c}{ Descripción de roles } \\
\hline Capitán & 1 & $\begin{array}{l}\text { Se encarga de elegir la mejor opción } \\
\text { de pedido teniendo en cuenta los } \\
\text { registros históricos. }\end{array}$ \\
$\begin{array}{c}\text { Sargento } \\
\text { primero }\end{array}$ & 1 & $\begin{array}{l}\text { Suministra los materiales necesarios } \\
\text { para que el capitán tome la decisión. } \\
\text { Se encarga de manejar las unidades de } \\
\text { comida en el mapa y de llenar todos los } \\
\text { formatos anexos. }\end{array}$ \\
\hline
\end{tabular}

Fuente: Autores

Además, para cumplir con la metodología explícita de realizar la lúdica, los creadores de esta adoptarán un rol de Pachamama o diosa de la naturaleza, que hará las veces de factor aleatorio del juego, al proveer, a través de una recreación teatral y de la ruleta presentada en la Figura 3, las diferentes adversidades y retrasos según el color asignado que se pueden presentar en la ruta diseñada para los jugadores.

\section{Materiales de la lúdica}

El primer recurso material necesario es la tabla de cálculo de la demanda, dispuesta en el formato anexo 1, en la cual se estima la demanda histórica de unidades de comida 
Jair Eduardo Rocha-González, Cristian David Quintero-Romero, Walter Stiven Santamaria-Melo Diseño de una actividad lúdica para enseñanza del Problema de Inventario de Una Sola Vez con Teoría de la Decisión: Abastece la tropa

que anteriormente otros pelotones han consumido en el mismo trayecto.

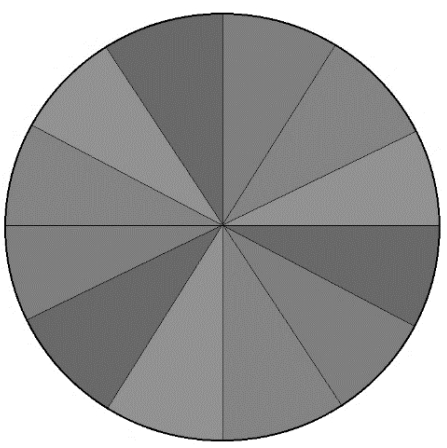

Figura 3. Imagen de la ruleta. Fuente: Autores.

Un segundo material requerido es una ruleta que está distribuida en doce franjas de cuatro colores alternados (rojo, naranja, amarillo y verde), donde cada color representa un número de días de retraso (ver Figura 3).

Los demás materiales necesarios están descritos por cantidad y tipo en la Tabla 5.

Tabla 5

Cantidad de cada material

\begin{tabular}{cc} 
Material & Cantidad para 5 mesas \\
\hline Tablero mapa & 5 \\
Tablero de asignación & 5 \\
Fichas de unidades de comida & 900 \\
Formatos de valor condicional & 5 \\
Fichas monedas de \$ 5 & 80 \\
Fichas monedas de \$3 & 80 \\
Formatos de registros históricos & 5 \\
\hline
\end{tabular}

Fuente: Autores.

Para lograr el propósito de la lúdica es necesario un espacio cómodo que garantice un flujo de materiales y personal alrededor de cinco mesas, cada una con tres sillas, más una silla adicional para el rol de Pachamama, Este espacio también puede contener una ubicación para algunos medios tecnológicos, tal como se muestra en la Figura 4.

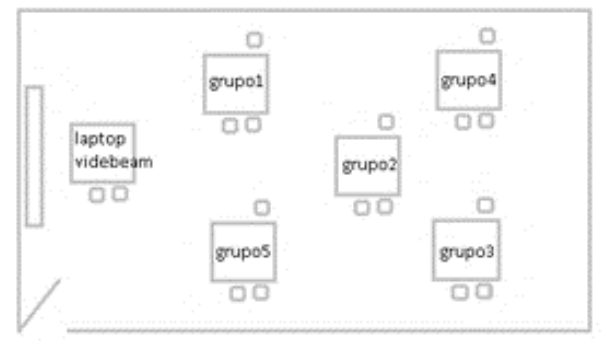

Figura 4. Plano distribución de equipos de trabajo. Fuente: Autores.

\section{Estructura de la lúdica}

En concordancia con los conceptos y los objetivos propuestos para la actividad lúdica, en términos de determinar la cantidad óptima a pedir en ambientes con demanda estocástica y producto con ciclo de vida o temporadas cortas, se presentan los siguientes pasos y contenidos didácticos. Además, en el formato anexo 2 se muestra el diagrama que explica el procedimiento de la lúdica.

De esta forma, el contexto en el cual se desenvuelve el escenario simulado tiene como ambiente el desplazamiento y relevo de personal militar en sitios alejados de la geografía colombiana, el cual es un proceso cotidiano para el Ejército Nacional. Usualmente, este proceso se realiza desde una base ubicada en un municipio desde el cual parten los relevos a otros sitios lejanos conectados a través de una ruta o camino de diferente naturaleza.

Por tanto, la recreación del escenario de esta actividad lúdica contará con el inicio del proceso en el municipio de San José del Guaviare, lugar que servirá de base para realizar el suministro y relevo de las tropas en el departamento del Guainía, en el extremo oriental de Colombia, a través de un desplazamiento por el rio Guaviare, donde la incertidumbre y el azar hará que los viajeros se retrasen y pocas veces puedan determinar de manera exacta la cantidad de comida a llevar para realizar el recorrido hasta Puerto Inírida, lugar meta del juego.

Ahora bien, la misión de cada equipo consiste en realizar el relevo de un pelotón regular que desde hace tiempo se encuentra establecido en un sitio, con la tarea de fortalecer la soberanía e institucionalidad del Estado en la región. De esta forma, los participantes del juego, organizados en diferentes equipos, tomarán el rol de grupo expedicionario que ha sido seleccionado para realizar el relevo de una de las tropas acuarteladas a lo largo del departamento del Guainía.

Para ello, los participantes en cada grupo tendrán roles que identifican un oficial de rango Capitán a cargo, un suboficial de rango Sargento y un grupo de soldados que tendrán como objetivo realizar la planeación logística del desplazamiento de los hombres, contemplando las posibles contingencias que puedan surgir en el camino y en el desplazamiento a través de transporte fluvial por el río Guaviare.

En este ámbito, la travesía diseñada cuenta con múltiples inconvenientes y situaciones que cada grupo expedicionario tendrá que sortear para avanzar en el juego en un tiempo determinado, estimando las cantidades de alimento adecuada para que todos los integrantes del grupo logren simular el recorrido en óptimo estado de salud. 
Jair Eduardo Rocha-González, Cristian David Quintero-Romero, Walter Stiven Santamaria-Melo

Diseño de una actividad lúdica para enseñanza del Problema de Inventario de Una Sola Vez con Teoría de la Decisión: Abastece la tropa

De esta manera, dos reglas fijadas para el desarrollo del juego establecen que, por cada día de viaje, la tropa de relevo consume cantidades de alimento diferentes, para las cuales se han fijado un costo de compra por unidad de tres unidades monetarias. Al ser consumidas, estas establecen un valor agregado que puede ser expresado como diez unidades monetarias, que representan el ahorro por medicamentos y otros aspectos que pueden presentarse por ausencia de alimentos.

Las decisiones que puede tomar cada grupo traerán como consecuencia dos posibles escenarios. En el primero, cada equipo puede comprar una cantidad inferior al consumo, lo cual representará una condición de faltantes. En el segundo, cada equipo podrá comprar una cantidad superior de alimentos al consumo, en cuyo caso tendrá sobrantes o excedentes.

Otras reglas que incorpora el juego establecen que, en caso de sobrar unidades de alimento, cada unidad puede ser vendida al final del viaje a un costo de una unidad monetaria por el alimento, más tres unidades monetarias de inversión por el transporte realizado, para un total de cuatro unidades monetarias por unidad sobrante. En el caso de una unidad faltante, esta costará el valor agregado o la utilidad que pudo haber tenido para la tropa transportada, es decir, diez unidades monetarias menos tres unidades de costo del alimento, para un total de siete unidades monetarias por unidad.

Una vez inicia el juego, las preguntas que cada grupo debe considerar son: ¿cuántas unidades de alimento su grupo sugiere comprar para el desplazamiento de la tropa? y ¿cuánto es el mínimo gasto que usted sugiere en relación con los faltantes o sobrantes de la campaña militar? Estos serán los interrogantes que tendrán que calcular para tomar decisiones de forma adecuada.

\section{Proceso para el desarrollo de la actividad lúdica}

Una vez establecidas las anteriores reglas de juego, se definen las etapas a seguir para su desarrollo con usuarios, divididas en pasos, para llevar a cabo la actividad lúdica de forma adecuada.

\section{Etapa I. Preparación de la actividad lúdica}

- Pasol.Caracterización de los roles de los organizadores para el desarrollo de la lúdica

El juego inicia cuando uno de los autores representa la incertidumbre y azar del recorrido con una representación teatral de la Pachamama, deidad de las culturas precolombinas que se asociaba con la respuesta de la naturaleza frente a la incursión del hombre en el ambiente natural.

Dicho autor seleccionado, mediante el papel descrito en el párrafo anterior, será el encargado de asignar a cada grupo una o varias condiciones que representan diversas adversidades a las cuales el grupo de participantes debe enfrentarse en la planeación y simulación del abastecimiento durante el simulacro del recorrido fluvial, tal como se realizaría en la realidad por parte del Ejercito Nacional.

De esta forma, se presenta una ficha de rol de este personaje en la Figura 5, donde se expresa el objetivo y acciones que debe realizar durante el juego.

\begin{tabular}{l} 
FICHA DE ROL \\
\hline PERSONAJE \\
\hline OBJETIVO: \\
\hline Se encarga de poseer y girar la ruleta para \\
identificar la adversidad con la cual se va a \\
enfrentar el pelotón durante su travesía, \\
asignando los días adicionales que la tropa \\
empleara en el recorrido normal de cuatro \\
días, además coloca en el mapa de cada \\
equipo las fichas de adversidad con la \\
información relevante del retraso y la causa \\
de este.
\end{tabular}

Figura 5. Ficha de rol "Pachamama”. Fuente: Autores.

- Paso II. Organización de los grupos de participantes

Se conformarán cinco grupos, cada uno con tres integrantes. Entre estos se elegirán a las personas que desempeñarán los roles de capitán, sargento y dragoneante en la actividad, de acuerdo y con la conciliación de voluntades de los integrantes de cada equipo, a quienes se dará una copia de la ficha de rol mostrada en la Figura 6.

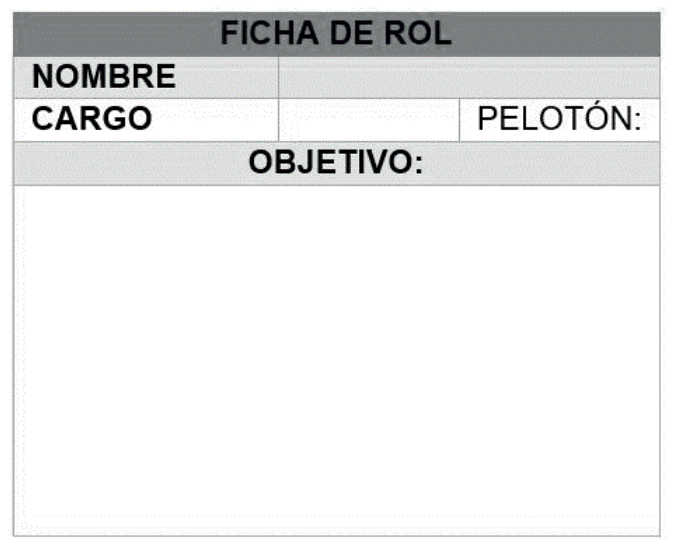

Figura 6. Ficha de rol genérica. Fuente: Autores.

Una vez conformados los grupos y conciliados los roles de capitán, sargento y dragoneante, cada uno de estos roles realizará las actividades y los objetivos descritos en las figuras 7, 8 y 9, respectivamente, para cada participante. 
Jair Eduardo Rocha-González, Cristian David Quintero-Romero, Walter Stiven Santamaria-Melo Diseño de una actividad lúdica para enseñanza del Problema de Inventario de Una Sola Vez con Teoría de la Decisión: Abastece la tropa

\begin{tabular}{|c|c|c|}
\hline \multicolumn{3}{|c|}{ FICHA DE ROL } \\
\hline NOMBRE & \multicolumn{2}{|l|}{$X X X X$} \\
\hline CARGO & CAPITÁN & PELOTÓN: X \\
\hline \multicolumn{3}{|c|}{ OBJETIVO: } \\
\hline \multicolumn{3}{|c|}{$\begin{array}{l}\text { Su rol implica cual es la cantidad de unidades } \\
\text { de alimento que debe llevar la tropa para } \\
\text { realizar la travesía, para ello tome en cuenta } \\
\text { los datos históricos de consumo de alimento } \\
\text { por otras tropas que han realizado este } \\
\text { recorrido. Comunique que cantidad de } \\
\text { alimento solicitar cuando le pregunten la } \\
\text { cantidad a pedir y gestione los recursos } \\
\text { monetarios para la compra. }\end{array}$} \\
\hline
\end{tabular}

Figura 7. Ficha de rol del capitán. Fuente: Autores.

\begin{tabular}{|c|c|c|}
\hline \multicolumn{3}{|c|}{ FICHA DE ROL } \\
\hline NOMBRE & $X X X X$ & \\
\hline CARGO & SARGENTO & PELOTÓN: $X$ \\
\hline \multicolumn{3}{|c|}{ OBJETIVO: } \\
\hline \multicolumn{3}{|c|}{$\begin{array}{l}\text { Su función en el equipo de trabajo es la de } \\
\text { analizar y determinar bajo un criterio la cantidad } \\
\text { de alimento a llevar. También se encarga de } \\
\text { entregar por cada día } 15 \text { unidades de comida a } \\
\text { la tropa. Este atento a las órdenes del capitán y } \\
\text { sugiera posibles criterios de pedido. Entregue } \\
\text { por cada día que pase en el juego la cantidad } \\
\text { de comida necesaria, explique y mantenga a la } \\
\text { tropa con alta moral ante posibles faltantes de } \\
\text { alimento. }\end{array}$} \\
\hline
\end{tabular}

Figura 8. Ficha de rol del sargento. Fuente: Autores.

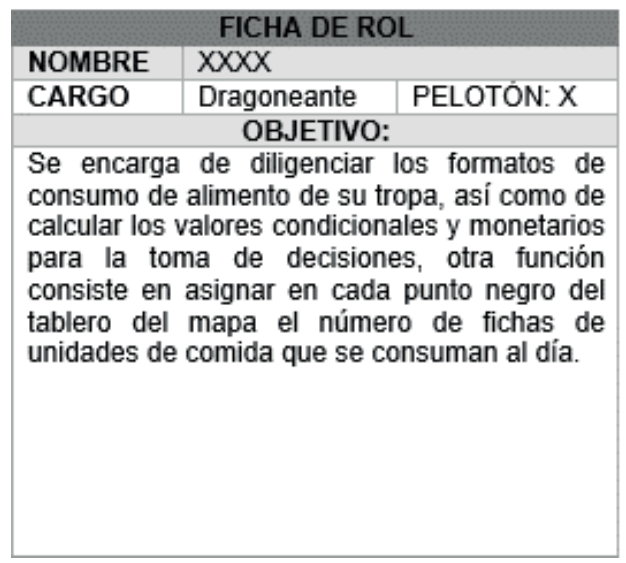

Figura 9. Ficha de rol del dragoneante. Fuente: Autores.

\section{- Paso III. Formalización del contexto}

Este paso comienza con la entrega de los formatos anexos, los cuales se definen como formato anexo 1: descripción de demanda histórica; formato anexo 3: cálculos de valores condicionales por combinación de pedidos y demanda probable, y formato Anexo 4: cálculo de valor monetario esperado.
De igual manera, cada grupo recibirá dieciséis monedas con denominación cinco e igual número de la denominación tres, para un total de treinta y dos monedas con ciento veintiocho unidades monetarias, un mapa del recorrido con puntos de reabastecimiento, las tres fichas de roles y el resumen del problema en el contexto diseñado.

- Paso IV. Determinación de la cantidad de días empleados en el viaje en ocasiones anteriores

La ruta plasmada en el mapa entregado a cada grupo presenta al río Guaviare en su recorrido entre las ciudades de San José del Guaviare y Puerto Inírida, recorrido que se realiza, de acuerdo con el contexto, en cuatro jornadas de travesía. Al final de cada una de ellas, se simboliza el lugar de arribo con una serie de puntos negros, donde a la orden de avance por cada jornada se hará girar la ruleta para representar los posibles percances o días de retraso para cada equipo, con lo que se logra una cantidad de quince registros diferentes, con el propósito de consolidar datos históricos.

Cada registro por equipo inicia con la simulación del desplazamiento de una ficha de lego que representa a la tropa. Esto se ejecuta sobre el mapa al primer punto negro del recorrido, donde se gira la ruleta y se obtiene uno de cuatro resultados posibles, según señale la ruleta, que son una demora de tres días al señalar el color rojo, dos días el color naranja, un día el color amarillo y ningún día el color verde, tal como se ilustra en la Figura 10. El color señalado en la ruleta se sumará al resultado de cuatro días, que es el mínimo de desplazamiento de la tropa para su relevo.

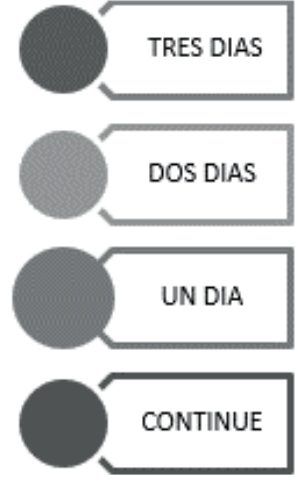

Figura 10. Semáforo de adversidades. Fuente: Autores.

Posteriormente, este proceso se repite con los siguientes tres puntos, para hallar la suma de días logrados como registro histórico, y así sucesivamente con cada jugador hasta el último, hasta llegar a una cifra considerable de registros que pueden ser consignados en el formato anexo 1 de descripción de demanda histórica.

Para entender este paso, considere que un jugador al girar la ruleta cuatro veces, por cada punto negro obtiene 
Jair Eduardo Rocha-González, Cristian David Quintero-Romero, Walter Stiven Santamaria-Melo

Diseño de una actividad lúdica para enseñanza del Problema de Inventario de Una Sola Vez con Teoría de la Decisión: Abastece la tropa

un resultado de un día en cada lugar, para un total de cuatro días, que al ser sumados con los cuatro días de recorrido normal da como resultado un trayecto de ocho días, valor que será usado como primer registro histórico y así sucesivamente con cada jugador hasta completar el formato anexo 1 de registro histórico, tomando como ejemplo la Tabla 6.

Tabla 6

Ejemplo del registro histórico

\begin{tabular}{cc}
\hline Número de días & Número de tropas históricas \\
\hline $4-6$ & \\
$7-9$ & \\
$10-12$ & \\
$13-16$ & \\
\hline
\end{tabular}

Fuente: Autores.

Una vez realizado este ejercicio múltiples veces, cada equipo tendrá disponible una colección de datos que representa las cantidades de días empleados en realizar diferentes viajes expresados en días, los cuales indican también el consumo de alimento por integrante del grupo en una proporción de una unidad de alimento por día, como se muestra en la Tabla 7.

Tabla 7

Ejemplo del registro histórico

\begin{tabular}{|c|c|}
\hline Número de días & Número de tropas históricas \\
\hline $4-6$ & IIIIIIII \\
\hline $7-9$ & ||||||||||||||||||| \\
\hline $10-12$ & IIII \\
\hline $13-16$ & $\||||| \mid$ \\
\hline
\end{tabular}

Fuente: Autores.

\section{Etapa II. Formulación del plan de abastecimiento de forma intuitiva}

- Paso I. Estimación de la probabilidad de duración en días del viaje

En esta modalidad del juego, el primer paso consiste en calcular la probabilidad de ocurrencia de duración de un intervalo de días. Para ello se realiza la suma de las líneas registradas por cada intervalo de días consignados en el Formato 1, con los cuales se calcula el cociente frente al total de datos de todos los intervalos de días obtenidos, de acuerdo con la ecuación (10).

\section{$N^{\circ}$ de días por intervalo Total de días realizados}

- Paso II. Cálculo de la cantidad de alimento a comprar de forma intuitiva
A partir de los cálculos de probabilidad de ocurrencia de días de viaje, y teniendo como base de cálculo que por cada día se consumen quince unidades de comida, se solicita a cada grupo diseñar un plan de compra y abastecimiento de la cantidad de alimento a llevar por cada tropa.

Para este caso, algunas simulaciones del escenario lúdico han definido que algunos jugadores determinan la mediana de la duración de los días y multiplican este resultado por quince unidades de alimento; es decir, diez días por quince unidades de alimento. En otros casos, establecen el punto medio del intervalo con mayor ocurrencia y a este valor le multiplican las quince unidades de alimento, mientras en otras ocasiones eligen comprar el máximo o el mínimo posible por diferentes razones de costo o satisfacción de la demanda, dejando de lado los costos marginales del caso.

- Paso III. Estimación del costo del plan de compra y abastecimiento

Para realizar la asignación de unidades de alimento, estas son representadas por una ficha redonda, la cual tiene el número quince, que hace referencia a unidades de comida, lo que implica que cada grupo puede comprar múltiplos de quince raciones como abastecimiento al distribuidor de alimento, que este caso será un integrante del grupo organizador.

Dependiendo de la cantidad de alimentos que cada grupo desea comprar para realizar el viaje, el participante con el rol de capitán realiza la compra de tantas unidades de alimento como hayan estimado consumir, realizando una transacción con las monedas y estimando el costo de compra de estas cantidades mediante la ecuación (11).

\section{Costo de compra para alimentos = Precio de compra \\ * Número de unidades de alimento (11)}

\section{- Paso IV. Realización de la simulación del viaje}

En esta etapa del juego cada grupo procede a realizar una simulación del viaje sobre el mapa, con una ficha que coloca sobre el origen, lugar en el cual se presenta la primera contingencia del viaje y que será representada por el personaje de la Pachamama, quien hace girar la ruleta para obtener un color y proporciona una ficha de adversidad que describe el evento y el número de días de retraso que tendrán por este evento, tal como se muestra en la Figura 11.

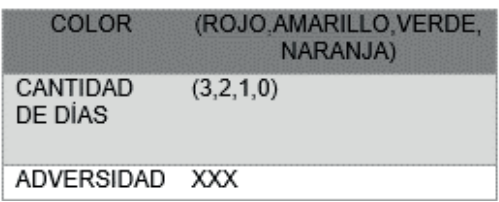

Figura 11. Tarjeta de tipo de adversidad genérica. Fuente: Autores. 
Jair Eduardo Rocha-González, Cristian David Quintero-Romero, Walter Stiven Santamaria-Melo Diseño de una actividad lúdica para enseñanza del Problema de Inventario de Una Sola Vez con Teoría de la Decisión: Abastece la tropa

Luego del proceso señalado, el grupo asignará en el mapa el número de unidades de alimento que requieren para sortear el recorrido en el nuevo tiempo fijado, descontando estas cantidades del pedido inicialmente comprado. Luego se realiza este procedimiento tres veces más sobre cada punto negro del mapa, hasta determinar la cantidad de alimentos a consumir en todo el viaje y si existen sobrantes o faltantes.

Cabe resaltar que la máxima cantidad de unidades de alimento que puede consumir la tropa es dieciséis unidades, así como la mínima cantidad de unidades de alimento es cuatro, puesto que el pelotón lo mínimo que puede gastar para hacer el trayecto es cuatro días.

La Figura 12 presenta el mapa de recorrido. En él se representan los puntos de origen, de destino y los posibles sitios intermedios de parada tras un día de recorrido sin percances que solventar.

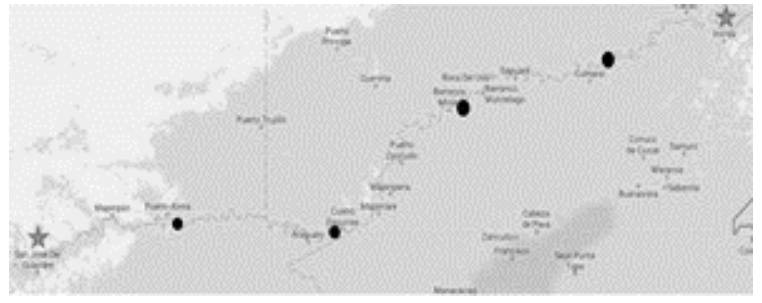

Figura 12. Mapa del recorrido. Fuente: Autores.

En relación con la forma en la cual aparecen los inconvenientes a resolver por el grupo participante, representados por la ruleta en poder del personaje de la Pachamama, estos podrán ser los descritos en la Tabla 8, donde se aprecia que en un giro de la ruleta es posible obtener uno de cuatro colores disponibles que representan diferentes problemas acaecidos que retrasan el viaje.

Tabla 8

Adversidades según el color

\begin{tabular}{ccc}
\hline Color & Adversidad & $\begin{array}{c}\text { Número de días } \\
\text { de retraso }\end{array}$ \\
\hline Rojo 1 & Daños del bote & 3 \\
Rojo 2 & Emboscada del enemigo & 3 \\
Rojo 3 & Se creció el rio & 3 \\
Naranja 1 & Se represó el rio & 2 \\
Naranja 2 & Tormenta & 2 \\
Naranja 3 & El río está minado & 2 \\
Amarillo 1 & Mordedura de una serpiente & 1 \\
Amarillo 2 & a un soldado & 1 \\
Amarillo 3 & Cocodrilos en el río & 1 \\
Verde 1 & Falla en el motor & 0 \\
Verde 2 & El día esta soleado & 0 \\
Verde 3 & Los están esperando lindas & mujeres \\
\hline
\end{tabular}

Fuente: Autores.
Al finalizar el recorrido y una vez se hayan puesto en el tablero del mapa todas las fichas de unidades de alimento requerido por todos los grupos participantes, se procede a analizar lo sucedido desde una perspectiva de unidades faltantes o sobrantes.

\section{Etapa III. Determinación de la cantidad de alimento a comprar mediante el uso de teoría de decisión}

\section{- Paso I. Fijación de la cantidad de días de viaje}

En este paso de la lúdica, se trae nuevamente la información acerca de los días empleados por tropas anteriores, desarrollado en el paso IV de la etapa I, para que, una vez realizado el nuevo método, los resultados pueden ser contrastables de forma paramétrica. Para ello, se copia esta información en el formato de valor condicional disponible en el anexo 3.

- Paso II. Estimación de las cantidades monetarias y de alimento por cada combinación demandada - pedido en el tablero de asignación

En este paso de la actividad lúdica, cada grupo recibirá dieciséis monedas de cinco unidades monetarias y la misma cantidad de monedas de tres unidades monetarias; además se distribuyen ochenta fichas de legos, cuya función será la representación de las unidades de alimento, en una proporción de una ficha por quince unidades de alimento.

Ahora, cada grupo inicia la actividad en el tablero de asignación, colocando en las columnas las posibles decisiones de pedido para almacenar, contabilizadas en cantidades de noventa, ciento cincuenta, ciento noventa y cinco y doscientos cuarenta unidades, que corresponden a la cantidad de unidades de alimento requeridas para realizar el recorrido simulado en seis, diez, trece y dieciséis días, que corresponden al valor máximo de cuatro intervalos de tiempo, tal como se estipuló en el formato anexo 1 realizado en la etapa II del juego, y reseñados en las Tablas 6 y 7 del documento.

En el mismo aspecto, en las filas del tablero de asignación, los grupos estipulan las posibles demandas que pueden ocurrir para los mismos valores superiores de los cuatro intervalos señalados en la Tabla 10; es decir, noventa, ciento cincuenta, ciento noventa y cinco y doscientos cuarenta unidades. De esta manera, se crea una matriz de demandas contra pedidos probables, en las cuales aparece un cuadro con tres divisiones en las que pueden ubicarse las fichas que representan las unidades de alimento, las monedas para la compra de unidades de alimento y las que representan el valor agregado para la tropa, tal como se muestra en la Tabla 9. 
Jair Eduardo Rocha-González, Cristian David Quintero-Romero, Walter Stiven Santamaria-Melo

Diseño de una actividad lúdica para enseñanza del Problema de Inventario de Una Sola Vez con Teoría de la Decisión: Abastece la tropa

Tabla 9

Descripción de una celda en el tablero de asignación

\begin{tabular}{|c|c|c|}
\hline Demanda & & $\begin{array}{c}\text { cena } \\
\text { ) }\end{array}$ \\
\hline$)$ & $\begin{array}{l}\text { Unidades de } \\
\text { alimento }\end{array}$ & $\begin{array}{c}\text { Valor agregado total } \\
\left(\mathrm{VA}_{\text {total }}\right) \\
\text { Costo de compra } \\
\text { total }\left(\mathrm{CC}_{\text {total }}\right)\end{array}$ \\
\hline
\end{tabular}

Fuente: Autores.

Para entender este paso, considere que es posible para cada grupo elegir comprar y almacenar noventa, ciento cincuenta, ciento noventa y cinco o doscientos cuarenta unidades de alimento, para ser consumidas durante el viaje, que tarda diez días, calculado a partir de los giros de la ruleta.

A partir de este tiempo la cantidad demandada de alimento, posterior al pedido, es de ciento cincuenta unidades, por lo cual en la celda de unidades de alimento se colocan diez unidades que representan esta cantidad.

De acuerdo con lo anterior, para realizar cada combinación de pedidos almacenados posible a realizar frente a la demanda ocurrida, el cálculo del costo total de compra ( de cada cantidad de pedido de alimentos para consumo de la tropa se realiza a través de la expresión 12:

$$
C C_{\text {Total }(i, j)}=A_{j} \times C
$$

Donde $c$ es el costo de compra por unidad de alimento.

De forma similar, el valor agregado total $\left(V A_{\text {Total(i.j) }}\right)$ es calculado como un valor de utilidad generada por la compra de una cantidad de alimentos. Para el caso anterior, esto se estima a través de la expresión 13:

$$
V A_{\text {Total }(i, j)}=\operatorname{Min}\left[A_{j} D_{j}\right] \times v a
$$

Donde va es el valor agregado que representa para la tropa tener disponible una unidad de alimento.

De esta manera, las cantidades de cada elemento a colocar en la matriz será la presentada en la Tabla 10, donde se describen las cantidades de fichas que representan las unidades de alimento, así como el costo total por compras y almacenamiento, y el valor agregado para consumo de alimentos por la tropa.

Tabla 10

\begin{tabular}{|c|c|c|c|c|c|c|c|c|}
\hline \multirow[t]{2}{*}{$\begin{array}{l}\text { Demanda } \\
\text { [raciones] }\end{array}$} & \multicolumn{2}{|c|}{$\begin{array}{c}\text { Almacena [raciones] } \\
\qquad(90)\end{array}$} & \multicolumn{2}{|c|}{$\begin{array}{l}\text { Almacena [raciones] } \\
\qquad(150)\end{array}$} & \multicolumn{2}{|c|}{$\begin{array}{l}\text { Almacena [raciones] } \\
\qquad(195)\end{array}$} & \multicolumn{2}{|c|}{$\begin{array}{c}\text { Almacena [raciones] } \\
\qquad(240)\end{array}$} \\
\hline & $\begin{array}{l}\text { Unidades de } \\
\text { alimentos } x \\
15 \text { raciones }\end{array}$ & $\begin{array}{c}\text { Valor } \\
\text { agregado } \\
\text { Costo compra }\end{array}$ & $\begin{array}{l}\text { Unidades de } \\
\text { alimentos } x \\
15 \text { raciones }\end{array}$ & $\begin{array}{c}\text { Valor } \\
\text { agregado } \\
\text { Costo compra }\end{array}$ & $\begin{array}{l}\text { Unidades de } \\
\text { alimentos } x \\
15 \text { raciones }\end{array}$ & $\begin{array}{c}\text { Valor } \\
\text { agregado } \\
\text { Costo compra }\end{array}$ & $\begin{array}{l}\text { Unidades de } \\
\text { alimentos } x \\
15 \text { raciones }\end{array}$ & $\begin{array}{c}\text { Valor } \\
\text { agregado } \\
\text { Costo compra }\end{array}$ \\
\hline \multirow[t]{2}{*}{ (90) } & 6 & 630 & 10 & 630 & 13 & 630 & 16 & 630 \\
\hline & & 270 & & 450 & & 585 & & 720 \\
\hline \multirow[t]{2}{*}{ (150) } & 6 & 630 & 10 & 1050 & 13 & 1050 & 16 & 1050 \\
\hline & & 270 & & 450 & & 585 & & 720 \\
\hline \multirow[t]{2}{*}{ (195) } & 6 & 630 & 10 & 1050 & 13 & 1365 & 16 & 1365 \\
\hline & & 270 & & 450 & & 585 & & 720 \\
\hline \multirow[t]{2}{*}{$(240)$} & 6 & 630 & 10 & 1050 & 13 & 1365 & 16 & 1680 \\
\hline & & 270 & & 450 & & 585 & & 720 \\
\hline
\end{tabular}

Tablero de asignación de valor agregado y costo de compra para diferentes almacenamientos

Fuente: Autores.

Estos cálculos de la utilidad esperada, conocida en teoría de la decisión como valor condicional, pueden ser escritos en el formato anexo 3 de valor condicional, verificando el análisis realizado de utilidad constante en el desarrollo de los cálculos para cada pedido almacenado con cantidades superiores, y de igual modo con las demandas realizadas.

\section{- Paso IV. Cálculo del valor monetario esperado}

En este paso se calcula la media de cada alternativa de decisión, que, para el caso, establece las cantidades a pedir y almacenar. Esta se logra al multiplicar cada valor condicional calculado en el paso anterior por la probabilidad de ocurrencia de la demanda para cada pedido y almacenaje posible, tal como se muestra en la ecuación 14, para posteriormente sumar los valores obtenidos por decisión que se pueda tomar.

$$
V M E=\sum_{i=1}^{n} V C_{i j} \times P\left(D_{i}\right)
$$

Donde:

$V C_{i j}$ : Valor condicional para cada combinación de pedido y almacenaje y demanda ocurrida.

$P(D)$ : Probabilidad de que suceda una cantidad de demanda. 
Jair Eduardo Rocha-González, Cristian David Quintero-Romero, Walter Stiven Santamaria-Melo Diseño de una actividad lúdica para enseñanza del Problema de Inventario de Una Sola Vez con Teoría de la Decisión: Abastece la tropa

- Paso V. Determinación de la mejor estrategia de pedido

Finalmente, se realiza el procedimiento descrito en la simulación del viaje, tal como se describió en la etapa II y en el paso IV, con la única variación de establecer como cantidad de pedido y almacenaje el valor hallado por medio del método de Valor Monetario Esperado del formato anexo 4; esto logra compararse con el método intuitivo y se encuentran diferencias significativas entre ambas maneras de cálculo.

Finalmente, se realiza un ejercicio de validación de la actividad lúdica respecto a la cohesión y la coherencia del contexto lúdico, con un grupo de estudiantes de ingeniería industrial y automatización, del semillero de investigación de una universidad de Bogotá. A partir de esta se identificó que los formatos establecidos para cada paso de la actividad deberían ser diligenciados y calculados por los participantes; del mismo modo, se reconocieron diferentes aportes que mejoraron la enseñanza de los conceptos a lo largo de las cinco sesiones realizadas.

En la primera sesión se obtuvieron argumentos por parte de los participantes donde se precisaban inquietudes sobre los conceptos de la proporción de las unidades o porciones de comida que se representaban en una ficha, para lo cual en la siguiente sesión se tuvo una mejor introducción sobre esta proporción y definición en los formatos entregados a los estudiantes.

Para la sesión dos se tuvo como retroalimentación explicar de manera más sencilla el costo por faltantes y sobrantes y el ratio de beneficio al tomar una buena decisión, para lo cual se tuvo más precisión en la explicación del costo por faltantes de una unidad de comida en el trayecto para la tropa y el costo por llevar unidades sobrantes en este.

En la sesión tres ya se expresaba un mejor entendimiento por parte de los participantes en la validación, pero para esta oportunidad se agregaron algunas situaciones exactas para los días de retraso y no solamente una abreviación. Esto significó un valor agregado para esta sesión.

Para terminar con los resultados obtenidos en la validación de coherencia y cohesión, para la cuarta sesión se tuvo un aporte sólido que facilitó la comprensión del formato anexo 4, al generar una tabla fácil de diligenciar y de mayor entendimiento para los participantes. Esto llevó a que en la última sesión realizada se obtuvieran buenos comentarios por parte de los estudiantes del semillero y se lograra comprobar la fluidez del juego diseñado.

\section{Conclusiones}

A través del desarrollo metodológico propuesto, fue posible obtener una actividad lúdica que puede ser útil para la enseñanza de la teoría de decisiones, a través de un caso de abastecimiento con productos de temporada, en el cual son fácilmente identificables los conceptos de Valor Monetario Esperado (VME), Probabilidad de Ocurrencia (PO) y Valor Condicional (VC).

Durante el desarrollo de la lúdica se presentaron diferentes retos, como la realización de la estructura metodológica escogida, puesto que dos de los autores la manejaban por primera vez, así como la escritura y coherencia del texto escrito durante el documento realizado y la consulta del estado del arte sobre la información relacionada, como uno de los últimos retos del ejercicio investigativo.

Esta actividad lúdica reseñada en su ejecución tendrá un bajo costo, debido a la utilización de materiales de fácil adquisición y valor asequible, en el cual se realizarán tres fases que pretenden tener dos objetivos de significancia frente a los conceptos de trabajo y satisfacción frente al juego, para contribuir a la gamificación de actividades de investigación de operaciones.

La metodología de cibernética de tercer orden empleada en el diseño tuvo un proceso de validación en el cual, a través de una serie de sesiones con estudiantes de ingeniería con conocimientos previos en la técnica, se pudieron identificar diferencias entre los propósitos de formación descritos en la metodología y el juicio de estos. De esta manera fue posible realizar un proceso de realimentación y mejora durante cada nueva sesión realizada.

La metodología propuesta proporciona a los participantes un escenario lúdico o gamificado de competencia, lo cual podría ser útil en la evaluación de competencias en el manejo de inventarios y toma de decisiones en el abastecimiento de productos de temporada (Chica Salgado, 2019), a través de un método de estudio de caso o juego de simulación en un salón de clase en un corto tiempo.

Como seguimiento de la investigación, se espera realizar la validación final de la lúdica en el VIII encuentro de la red IDDEAL, donde se podrá contrastar la enseñanza de esta didáctica en los participantes. Por otra parte, se espera realizar otro juego de la misma temática con una metodología distinta para identificar el alcance de cada una de estas en la enseñanza del tema. 
Jair Eduardo Rocha-González, Cristian David Quintero-Romero, Walter Stiven Santamaria-Melo

Diseño de una actividad lúdica para enseñanza del Problema de Inventario de Una Sola Vez con Teoría de la Decisión: Abastece la tropa

\section{Referencias}

Arango Londoño, C. A., Rocha González, J. E. y Pinzón Rueda, W. A. (2014). Diseño de lúdicas usando circuito de cibernética de primer orden. 12th Latin American and Caribbean Conference for Engineering and Technology, 1-10. http://www.laccei.org/ LACCEI2014-Guayaquil/RefereedPapers/RP103.pdf

Bierman, H., Bonini, C. P. y Hausman, W. H. (1996). Analisis cuantitativo para la toma de decisiones. McGraw-Hill.

Chica Salgado, C. A. (2019). Modelo matemático de programación por metas para coadyuvar a la toma de decisiones en la selección de alternativas de inversión en Pymes. I+D Revista de Investigaciones, 13(1), 47-56. https://doi.org/10.33304/revinv.v13n12019005

Nevison, C. y Burstein, M. (1984). The Dynamic Lot-Size Model with Stochastic Lead Times. Management Science, 30(1), 100-109. https://doi.org/10.1287/ mnsc.30.1.100

Nova Arevalo, N. A., Pinzón Rueda, W. A. y Quintero, R. (2011). Cibernética de tercer orden y su aplicación a la telefonía móvil.

Richards, F. R. (1976). A Stochastic Model of a RepairableItem Inventory System with Attrition and Random Lead Times. Operations Research, 24(1), 118-130. https://doi.org/10.1287/opre.24.1.118

Rocha-Gonzalez, J. (2011). Propuesta metodológica para la implementación de la lúdica como alternativa en la enseñanza en ingeniería industrial. Tercer Seminario Internacional Sistemas Productivos SISPRO: La Lúdica en la Enseñanza de la Ingenieria Industrial.

Rocha González, J. E., Arango, C. A., Mejía Ospina, L. A. y Pinzón Rueda, W. A. (2014). Diseño y evaluacion de juegos, dispoitivos lúdicos, pedagogicos y didacticos. Una propuesta desde la cibernetica de tercer orden.

Rocha González, J. E., Arango Londoño, C. A. y Pinzón Rueda, W. A. (2015). Lúdicas diseñadas como didáctica usando cibernética de tercer orden. Proceedings of the 13th Latin American and Caribbean Conference for Engineering and Technology Engineering Education Facing the Grand Challenges: What Are We Doing? https://doi.org/10.18687/ LACCEI2015.1.1.216

Silver, E., Pike, D. y Peterson, R. (1998). Inventory Management and Production Planning and Scheduling (3. ${ }^{\text {a }}$ ed.). Wiley.
Sippper, D. y Bulfin, R. L. (1998). Planeación y control de la producción. McGraw-Hill.

Sphicas, G. P. (1982). On the Solution of an Inventory Model with Variable Lead Times. Operations Research, 30(2), 404-410. https://doi.org/10.1287/ opre.30.2.404

Tersine, R. J. (1998). Principles of Inventory and Materials Management (4. ${ }^{\mathrm{a}}$ ed.). Prentice Hall.

VanMieghem,J.A.y Rudi, N.(2002). NewsvendorNetworks: Inventory Management and Capacity Investment with Discretionary Activities. Manufactururing and Service Operations Management, 4(4), 313-335.

Xue, X., Shen, Q., Tan, Y., Zhang, Y. y Fan, H. (2011). Comparing the Value of Information Sharing Under Different Inventory Policies in Construction Supply Chain. International Journal of Project Management, 29(7), $\quad$ 867-876. https://doi.org/10.1016/j. ijproman.2011.04.003 
Jair Eduardo Rocha-González, Cristian David Quintero-Romero, Walter Stiven Santamaria-Melo Diseño de una actividad lúdica para enseñanza del Problema de Inventario de Una Sola Vez con Teoría de la Decisión: Abastece la tropa

Anexo 1. Cálculo de la demanda

\begin{tabular}{|l|l|l|}
\hline Número de días & Numero de pelotones & Probabilidad de ocurrencia \\
\hline & & \\
\hline & & \\
\hline & & \\
\hline & & \\
\hline
\end{tabular}

Con la siguiente ecuación se debe calcular la probabilidad de ocurrencia:

$N^{\circ}$ de ocurrencias de duración por intervalo

Total de ocurrencias por días realizados

¿Qué cantidad de unidades de comida desea comprar su tropa?

¿Cuántas unidades de alimento su grupo sugiere comprar para el desplazamiento de la tropa?

¿Cuánto es el mínimo gasto que usted sugiere en relación con los faltantes o sobrantes de la tropa?

\section{Anexo 2. Diagrama de Flujo}

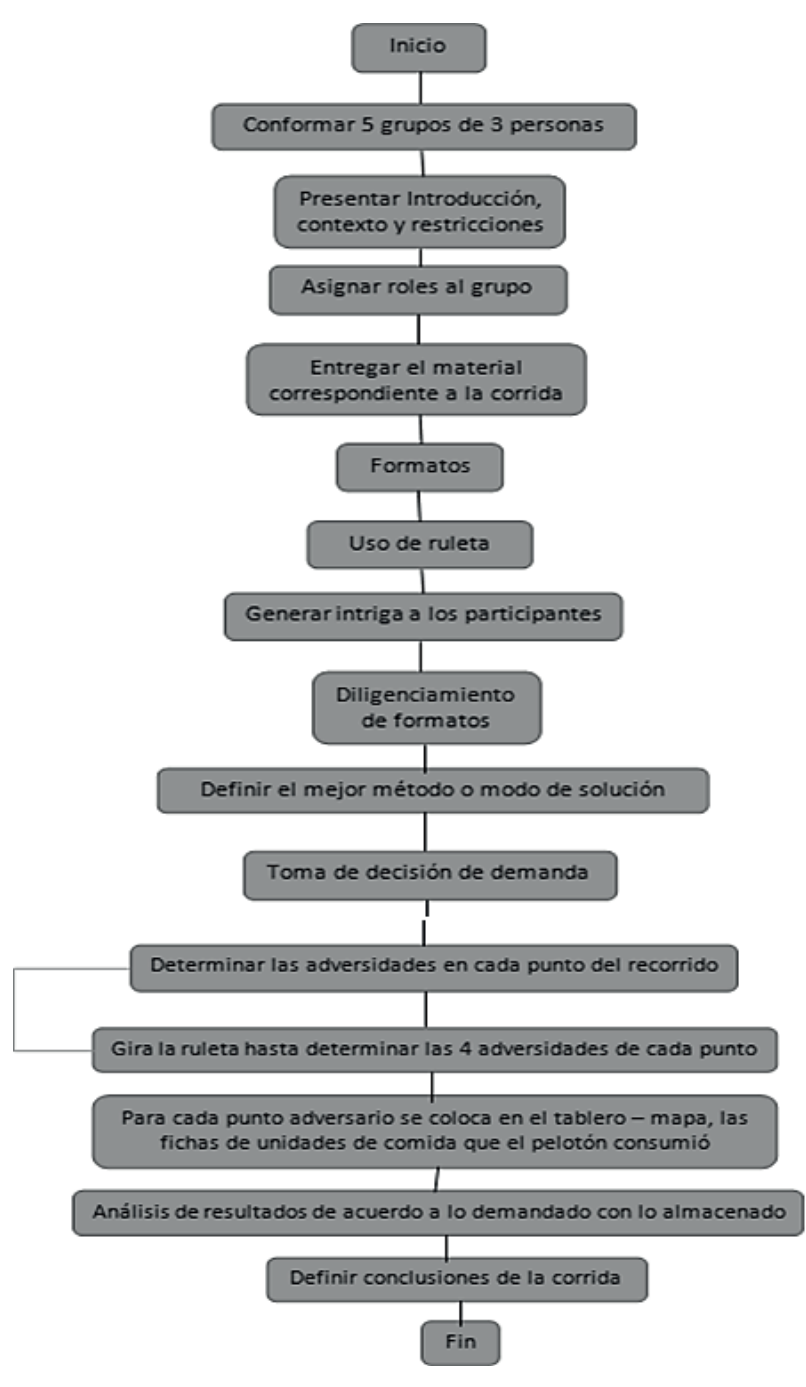


Jair Eduardo Rocha-González, Cristian David Quintero-Romero, Walter Stiven Santamaria-Melo

Diseño de una actividad lúdica para enseñanza del Problema de Inventario de Una Sola Vez con Teoría de la Decisión: Abastece la tropa

Anexo 3. Formato de valor condicional

\begin{tabular}{|c|c|c|c|c|}
\hline Demanda & Almacena 90 & Almacena 150 & Almacena 195 & Almacena 240 \\
\hline 90 & & & & \\
\hline 150 & & & & \\
\hline 195 & & & & \\
\hline 240 & & & & \\
\hline
\end{tabular}

Llene el tablero de asignación con las siguientes fórmulas

$$
\begin{gathered}
C C_{\text {Total }(i, j)}=\mathrm{A} \times \times C \\
V A_{\text {Total( }(, j))}=\operatorname{Min}[A j, D j] \times v a
\end{gathered}
$$

\begin{tabular}{|c|c|c|c|}
\hline Dem & nda & \multicolumn{2}{|c|}{ Almacena ( ) } \\
\hline \multirow{2}{*}{(} & \multirow{2}{*}{ ) } & \multirow{2}{*}{ Unidades de alimento } & Valor agregado total (VA total) \\
\hline & & & Costo de compra total (CC total) \\
\hline
\end{tabular}

Para esto se les muestra el siguiente ejemplo.

Teniendo claro y totalmente diligenciado el tablero de asignación llene el anexo con la siguiente fórmula

$$
\text { Valor condicional }=V C_{i j}=\text { Utilidad }=V A_{\text {Total }(i, j)}-C C_{\text {Total( }(i, j)}
$$

\begin{tabular}{|c|c|c|c|c|c|c|c|c|c|}
\hline \multirow[b]{2}{*}{ Demanda } & \multirow[b]{2}{*}{$\begin{array}{c}\text { Probabilidad } \\
\text { del suceso }\end{array}$} & \multicolumn{2}{|c|}{ ( Almacena } & \multicolumn{2}{|c|}{ Almacena } & \multicolumn{2}{|c|}{ ( Almacena } & \multicolumn{2}{|c|}{ ( Almacena } \\
\hline & & $\begin{array}{c}\text { Valor } \\
\text { condicional }\end{array}$ & $\begin{array}{c}\text { VC } \\
\text { ponderado } \\
\text { por la } \\
\text { probabilidad } \\
\text { del suceso }\end{array}$ & $\begin{array}{c}\text { Valor } \\
\text { condicional }\end{array}$ & $\begin{array}{c}\text { VC } \\
\text { ponderado } \\
\text { por la } \\
\text { probabilidad } \\
\text { del suceso }\end{array}$ & $\begin{array}{c}\text { Valor } \\
\text { condicional }\end{array}$ & $\begin{array}{c}\text { VC } \\
\text { ponderado } \\
\text { por la } \\
\text { probabilidad } \\
\text { del suceso }\end{array}$ & $\begin{array}{c}\text { Valor } \\
\text { condicional }\end{array}$ & $\begin{array}{c}\text { VC } \\
\text { ponderado } \\
\text { por la } \\
\text { probabilidad } \\
\text { del suceso }\end{array}$ \\
\hline$(1)$ & & & & & & & & & \\
\hline() & & & & & & & & & \\
\hline() & & & & & & & & & \\
\hline() & & & & & & & & & \\
\hline Valor Mon & o Esperado & & & & & & & & \\
\hline
\end{tabular}

Anexo 4. Cálculos de valores monetarios esperados.

Para calcular el Valor Monetario Esperado por la probabilidad del suceso aplique la siguiente fórmula:

$$
V M E=\sum_{i=1}^{n} V C_{i j} \times P\left(D_{i}\right)
$$

Donde:

$V C_{i j}$ : Valor condicional para cada combinación de pedido y almacenaje y demanda ocurrida.

$P\left(D_{j}\right)$ : Probabilidad de que suceda una cantidad de demanda.

¿Cuál es su concepto de la lúdica? ¿Recomendaciones? 